Bull. Korean Math. Soc. 50 (2013), No. 6, pp. 1887-1903

http://dx.doi.org/10.4134/BKMS.2013.50.6.1887

\title{
MCCOY CONDITION ON IDEALS OF COEFFICIENTS
}

\author{
Jeoung Soo Cheon, Chan Huh, Tai Keun Kwak, and Yang Lee
}

\begin{abstract}
We continue the study of McCoy condition to analyze zerodividing polynomials for the constant annihilators in the ideals generated by the coefficients. In the process we introduce the concept of ideal- $\pi$ McCoy rings, extending known results related to McCoy condition. It is shown that the class of ideal- $\pi$-McCoy rings contains both strongly McCoy rings whose non-regular polynomials are nilpotent and 2-primal rings. We also investigate relations between the ideal- $\pi$-McCoy property and other standard ring theoretic properties. Moreover we extend the class of ideal- $\pi$-McCoy rings by examining various sorts of ordinary ring extensions.
\end{abstract}

\section{Ideal- $\pi$-McCoy rings}

Throughout this note every ring is associative with identity unless otherwise stated. Let $R$ be a ring and we use $R[x]$ to denote the polynomial ring with an indeterminate $x$ over $R$. Denote the $n$ by $n$ full matrix ring over $R$ by $\operatorname{Mat}_{n}(R)$ and the $n$ by $n$ upper (resp. lower) triangular matrix ring over $R$ by $U_{n}(R)$ (resp. $\left.L_{n}(R)\right)$. Use $E_{i j}$ for the matrix with $(i, j)$-entry 1 and elsewhere $0 . \mathbb{Z}$ and $\mathbb{Z}_{n}$ denote the set of integers and the ring of integers modulo $n$, respectively. Note $\operatorname{Mat}_{n}(R)[x] \cong \operatorname{Mat}_{n}(R[x])$ and $U_{n}(R)[x] \cong U_{n}(R[x])$. We will apply these isomorphisms freely. $N_{*}(R)$ and $N(R)$ denote the prime radical and the set of all nilpotent elements in $R$, respectively.

McCoy [21, Theorem 2] showed the following fact in 1942:

$$
f(x) g(x)=0 \text { implies } f(x) r=0 \text { for some nonzero } r \in R,
$$

where $f(x)$ and $0 \neq g(x)$ are polynomials over a commutative ring $R$. Based on this result, Nielsen [22] in 2006 called a (possibly noncommutative) ring $R$ (possibly without identity) right McCoy when the equation $f(x) g(x)=0$ implies $f(x) r=0$ for some nonzero $r \in R$, where $f(x), 0 \neq g(x)$ are polynomials in $R[x]$. Left McCoy rings are defined similarly. If a ring is both left and right McCoy, then the ring is called a McCoy ring. Nielsen [22, Section 3 and Section 4] showed that the McCoy condition is not left-right symmetric.

Received April 10, 2012; Revised April 19, 2013.

2010 Mathematics Subject Classification. 16D25, 16 S36.

Key words and phrases. ideal- $\pi$-McCoy ring, strongly McCoy ring, $\pi$-McCoy ring, polynomial ring, matrix ring, the classical right quotient ring. 
It is well-known that the set of nilpotent elements forms an ideal in any commutative ring. This property is also possessed by the following kinds of noncommutative rings. Given a ring $R$, Shin [24, Proposition 1.11] proved that $N_{*}(R)=N(R)$ if and only if every minimal prime ideal of $R$ is completely prime. Birkenmeier et al. [6] called a ring $R$ 2-primal when $N_{*}(R)=N(R)$. Note that $R$ is 2-primal if and only if $R / N_{*}(R)$ is reduced. A well-known property between the commutativity and the 2-primal condition is the insertionof-factors-property (or simply IFP). Due to Bell [5], a ring $R$ is called IFP if $a b=0$ implies $a R b=0$ for $a, b \in R$. IFP rings are 2-primal by [24, Theorem 1.5]. Following Cohn [8], a ring $R$ is called reversible if $a b=0$ implies $b a=0$ for $a, b \in R$. Anderson and Camillo [2], observing the rings whose zero products commute, used the term $Z C_{2}$ for what is called reversible. A ring $R$ is called reduced if $N(R)=0$. Reduced rings are reversible via a simple computation, and commutative rings are clearly reversible. Every reversible ring is McCoy by [22, Theorem 2], and hence both commutative rings and reduced rings are McCoy. It is evident that reversible rings are IFP. However IFP rings need not be right McCoy by [22, Section 3]. There exist right McCoy rings that are not IFP with the help of [19, Theorem 2] and [17, Example 1.3].

According to Hong et al. [12], a ring $R$ (possibly without identity) is called strongly right $M c$ Coy provided that $f(x) g(x)=0$ implies $f(x) r=0$ for some nonzero $r$ in the right ideal of $R$ generated by the coefficients of $g(x)$, where $f(x)$ and $g(x)$ are nonzero polynomials in $R[x]$. Strongly left McCoy rings are defined similarly. If a ring is both strongly left and strongly right McCoy, then the ring is called a strongly McCoy ring. Reversible rings are strongly McCoy by [12, Theorem 1.6] or the proof of Nielsen [22, Theorem 2] and strongly McCoy rings are clearly McCoy. A ring is usually called right duo if each right ideal is two-sided. It is easily checked that right duo rings are IFP. Right duo rings are strongly right McCoy by the proof of [7, Theorem 8.2], and moreover we can find more concrete shape of right annihilators in the proof of $[12$, Theorem 1.11].

Due to Jeon et al. [15], a ring $R$ (possibly without identity) is called $\pi$-McCoy if $f(x) g(x) \in N(R[x])$ implies $f(x) r \in N(R[x])$ for some nonzero $r \in R$, where $f(x)$ and $g(x)$ are nonzero polynomials in $R[x]$. The class of $\pi$-McCoy rings contains McCoy rings by [15, Proposition 1.1(2)] and 2-primal rings (hence reversible rings, right duo rings and IFP rings) by [15, Proposition 1.7]. Next we consider the $\pi$-McCoy in conjunction with the nilpotency.

Let $R$ be a ring and $f(x), 0 \neq g(x) \in R[x]$ and $J$ be the ideal of $R$ generated by the coefficients of $g(x)$. In this situation consider the following condition:

(*) $\quad f(x) g(x) \in N(R[x])$ implies $f(x) a \in N(R[x])$ for some nonzero $a \in J$.

Recall that an element is called right (resp. left) regular if its right (resp. left) annihilator is zero. An element is called regular if it is both left and right regular. 
Proposition 1.1. (1) The condition $(*)$ is left-right symmetric.

(2) Let $R$ be a ring over which non-regular polynomials are nilpotent. If $R$ is strongly McCoy, then $R$ satisfies the condition (*).

Proof. (1) Let $R$ be a ring satisfying the condition $(*)$, and we will show that $R$ satisfies the left version of the condition $(*)$. Say $f(x) g(x) \in N(R[x])$ for $f(x), g(x) \in R[x]$ with $f(x) \neq 0$. From $f(x) g(x) \in N(R[x])$ we also get $g(x) f(x) \in N(R[x])$. Since $R$ satisfies the condition $(*), g(x) b \in N(R[x])$ for some nonzero $b$ in the ideal of $R$ generated by the coefficients of $f(x)$. This yields $b g(x) \in N(R[x])$. The converse can be proved by changing the roles of $f(x)$ and $g(x)$.

(2) Suppose that $R$ is a strongly McCoy ring whose non-regular polynomials are nilpotent. Let $f(x) g(x) \in N(R[x])$ for $f(x), g(x) \in R[x]$. If $f(x)=0$ or $g(x)=0$, then $R$ satisfies both the condition $(*)$ and the left version of the condition $(*)$. So we suppose that $f(x) \neq 0$ and $g(x) \neq 0$. We apply the proof of [15, Proposition 1.1(2)]. Let $(f(x) g(x))^{n}=0$ and $(f(x) g(x))^{n-1} \neq 0$ for some $n \geq 1$. Let $I$ and $J$ be the ideals of $R$ generated by the coefficients of $f(x)$ and $g(x)$, respectively.

Case 1. $f(x) g(x)=0$ and $g(x) f(x)=0$

Since $R$ is strongly McCoy, there exist $0 \neq a \in J$ and $0 \neq b \in I$ such that $f(x) a=0$ and $b g(x)=0$.

Case 2. $f(x) g(x)=0$ and $g(x) f(x) \neq 0$

Since $R$ is strongly McCoy, there exist $0 \neq a \in J$ and $0 \neq b \in I$ such that $f(x) a=0$ and $b g(x)=0$.

Case 3. $f(x) g(x) \neq 0$ and $g(x) f(x)=0$

Since $R$ is strongly McCoy, there exist $0 \neq a \in J$ and $0 \neq b \in I$ such that $a f(x)=0$ (hence $f(x) a \in N(R[x])$ ) and $g(x) b=0$ (hence $b g(x) \in N(R[x])$ ).

Case 4. $f(x) g(x) \neq 0$ (then $n \geq 2$ ) and $g(x) f(x) \neq 0$

Recall $(f(x) g(x))^{n-1} \neq 0$. From $(f(x) g(x))(f(x) g(x))^{n-1}=0$, there exists $0 \neq b \in I \cap J$ such that $f(x) g(x) b=0$ since $R$ is strongly right McCoy. So $g(x) b \in N(R[x])$ by the hypothesis, entailing $b g(x) \in N(R[x])$.

By Cases 1, 2, 3 and 4, we have that

$$
b g(x) \in N(R[x]) \text { for some } 0 \neq b \in I .
$$

Thus $R$ satisfies the condition (*) by (1).

As we see in the proof of Proposition 1.1(2), the condition $(*)$ is satisfied automatically when $f(x)=0$ or $g(x)=0$. So we will examine the condition $(*)$, assuming that $f(x) \neq 0$ and $g(x) \neq 0$.

The strongly McCoy condition is not left-right symmetric by help of $[16$, Example 1.8]. Based on Proposition 1.1, a ring will be called ideal- $\pi-M c C o y$ if it satisfies the condition $(*)$. Ideal- $\pi$-McCoy rings are clearly $\pi$-McCoy but 
the converse need not hold by Example 1.4(3) below. Note that there exists an ideal- $\pi$-McCoy ring which is not strongly McCoy by Example 1.3(2),(3) to follow.

Given a ring $R$ and $n \geq 2$, consider the subrings $D_{n}(R)=\left\{\left(m_{i j}\right) \in\right.$ $\left.U_{n}(R) \mid m_{11}=\cdots=m_{n n}\right\}$ and $V_{n}(R)=\left\{m=\left(m_{i j}\right) \in D_{n}(R) \mid m_{s t}=\right.$ $m_{(s+1)(t+1)}$ for $s=1, \ldots, n-2$ and $\left.t=2, \ldots, n-1\right\}$ of $U_{n}(R)$. For any set $M$ of matrices over a ring $R, M^{T}$ denotes the set of all transposes of matrices in $M$.

Lemma 1.2. (1) Let $R$ be a ring with an essential ideal $I$ of $R$ such that $I[x] \subseteq N(R[x])$. Then $R$ is ideal- $\pi-M c$ Coy.

(2) Let $R$ be a ring with a nilpotent essential ideal. Then $\operatorname{Mat}_{n}(R)$ is ideal$\pi-M c$ Coy for $n \geq 1$.

(3) Let $R$ be a ring with an essential ideal whose finitely generated subrings are nilpotent. Then $\operatorname{Mat}_{n}(R)$ is ideal- $\pi-M c$ Coy for $n \geq 1$.

(4) Both $U_{n}(A)$ and $L_{n}(A)$ are ideal- $\pi-M c$ Coy for any ring $A$ and $n \geq 2$.

(5) Both $D_{n}(A)$ and $D_{n}(A)^{T}$ are ideal- $\pi-M c$ Coy for any ring $A$ and $\bar{n} \geq 2$.

(6) Both $V_{n}(A)$ and $V_{n}(A)^{T}$ are ideal- $\pi-M c C o y$ for any ring $A$ and $n \geq 2$.

(7) The factor ring $R[x] /\left\langle x^{n}\right\rangle$ is ideal- $\pi-M c$ Coy for any $n \geq 2$, where $\left\langle x^{n}\right\rangle$ is a two-sided ideal of $R[x]$ generated by $x^{n}$.

(8) Let $A, B$ be any rings and ${ }_{A} M_{B}$ be an $(A, B)$-bimodule such that $a M \neq 0$ and $M b \neq 0$ for $0 \neq a \in A$ and $0 \neq b \in B$. Then $\left(\begin{array}{cc}A & M \\ 0 & B\end{array}\right)$ is ideal- $\pi-M c C o y$.

Proof. (1) Let $f(x) g(x) \in N(R[x])$ for nonzero polynomials $f(x), g(x)$ in $R[x]$ and let $J$ be the ideal of $R$ generated by the coefficients of $g(x)$. Since $I$ is an essential ideal, we have $I \cap J \neq 0$. If $f(x) \in(I \cap J)[x]$, then $f(x) r \in N(R[x])$ for all $r \in R$. If $f(x) \notin(I \cap J)[x]$, then $f(x) s \in N(R[x])$ for all nonzero $s \in I \cap J$. Thus $R$ is ideal- $\pi$-McCoy.

(2) Let $I$ be a nilpotent essential ideal of $R$. Then $\operatorname{Mat}_{n}(I)$ is a nilpotent essential ideal of $\operatorname{Mat}_{n}(R)$. Clearly $\operatorname{Mat}_{n}(I)[x] \subseteq N\left(\operatorname{Mat}_{n}(R)[x]\right)$. Thus $\operatorname{Mat}_{n}(R)$ is ideal- $\pi$-McCoy by (1).

(3) Let $I$ be an essential ideal whose finitely generated subrings are nilpotent. Then $\operatorname{Mat}_{n}(I)$ is also an essential ideal of $\operatorname{Mat}_{n}(R)$. Let $f(x)=\left(a(0)_{i j}\right)+$ $\left(a(1)_{i j}\right) x+\cdots+\left(a(n)_{i j}\right) x^{n} \in \operatorname{Mat}_{n}(I)[x]$ and $S$ be the subring of $I$ generated by $a(k)_{i j}$ 's for $k=0, \ldots, n$ and $i, j=1, \ldots, n$. By hypothesis, $S$ is nilpotent and this yields that $f(x)$ is nilpotent. Thus $\operatorname{Mat}_{n}(R)$ is ideal- $\pi$-McCoy by (1).

(4) Let $A$ be any ring and $R=U_{n}(A)$ for $n \geq 2$. Then

$$
I=\left\{\left(m_{i j}\right) \in R \mid m_{i i}=0 \text { for all } i=1, \ldots, n\right\}
$$

is an essential ideal of $R$ such that $I^{n}=0$. Thus $R$ is ideal- $\pi$-McCoy by (2).

The proofs of $(5),(6),(8)$ are almost same as (4), noting that $\left(\begin{array}{ll}0 & M \\ 0 & 0\end{array}\right)$ is a nilpotent essential ideal of $\left(\begin{array}{cc}A & M \\ 0 & B\end{array}\right)$.

$(6) \Leftrightarrow(7)$ follows from the well-known fact that $V_{n}(R) \cong R[x] /\left\langle x^{n}\right\rangle$. 
Question. Is a ring $R$ ideal- $\pi$-McCoy when $\operatorname{Mat}_{n}(R)\left(U_{n}(R)\right)$ is ideal- $\pi$ McCoy?

Given a ring $R$ and an $(R, R)$-bimodule $M$, the trivial extension of $R$ by $M$ is the $\operatorname{ring} T(R, M)=R \oplus M$ with the usual addition and the following multiplication: $\left(r_{1}, m_{1}\right)\left(r_{2}, m_{2}\right)=\left(r_{1} r_{2}, r_{1} m_{2}+m_{1} r_{2}\right)$. This is isomorphic to the ring of all matrices $\left(\begin{array}{cc}r & m \\ 0 & r\end{array}\right)$, where $r \in R$ and $m \in M$ and the usual matrix operations are used.

Hence, the trivial extension $T(R, R)$ of any $\operatorname{ring} R$ is an ideal- $\pi$-McCoy ring by the similar argument to Lemma 1.2(4).

We will use freely the fact that ideal- $\pi$-McCoy rings are $\pi$-McCoy.

Example 1.3. (1) Let $R$ be a reduced ring. Then $\operatorname{Mat}_{n}(R)$ is not $\pi$-McCoy

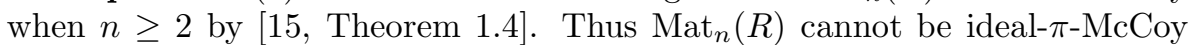
over a reduced ring $R$.

(2) There exist many kinds of rings that satisfy the hypothesis of Lemma $1.2(2)$. For example, $\mathbb{Z}_{p^{m}}(m \geq 2)$ for a prime $p$ has an essential nilpotent ideal $p \mathbb{Z}_{p^{m}}$. So $R=\operatorname{Mat}_{n}\left(\mathbb{Z}_{p^{m}}\right)(n \geq 1, m \geq 2)$ is ideal- $\pi$-McCoy by Lemma 1.2(2). Note that $R$ is neither left nor right McCoy by [15, Proposition 1.6] and so $R$ is not strongly McCoy.

(3) By Lemma 1.2(4), we can always construct an ideal- $\pi$-McCoy ring that is neither left nor right McCoy. $U_{n}(A)$ (for any $\operatorname{ring} A$ and $n \geq 2$ ) is ideal- $\pi$ McCoy but neither left nor right McCoy by [15, Example 1.3] and hence $R$ is not strongly McCoy.

Recall that the class of $\pi$-McCoy rings contains McCoy rings and ideal- $\pi$ McCoy rings. Moreover the properties of the McCoy and the ideal- $\pi$-McCoy are independent of each other by the following.

Example 1.4. (1) (non-semiprime case) There exist ideal- $\pi$-McCoy rings that are not one-sided McCoy by Example 1.3(2),(3).

(2) (semiprime case) There exists an ideal- $\pi$-McCoy ring that is not right McCoy, applying the proof of [14, Theorem 2.2]. Let $S$ be a reduced ring, $n$ be a positive integer and $R_{n}$ be the $2^{n}$ by $2^{n}$ upper triangular matrix ring over $S$. Define a map $\sigma: R_{n} \rightarrow R_{n+1}$ by $A \mapsto\left(\begin{array}{cc}A & 0 \\ 0 & A\end{array}\right)$, then $R_{n}$ can be considered as a subring of $R_{n+1}$ via $\sigma$ (i.e., $A=\sigma(A)$ for $A \in R_{n}$ ). Notice that $D=\left\{R_{n}, \sigma_{n m}\right\}$, with $\sigma_{n m}=\sigma^{m-n}$ whenever $n \leq m$, is a direct system over $\{1,2, \ldots\}$. Set $R=\lim R_{n}$ be the direct limit of $D$. Then $R$ is a semiprime ring by [14, Theorem 2.2], and $R$ is neither left nor right McCoy by [15, Example 1.3]. Moreover $R$ is an ideal- $\pi$-McCoy ring by Theorem 2.3 to follow since every $R_{n}$ is ideal- $\pi$-McCoy by Lemma $1.2(4)$. As another proof, consider the ideal $I=\{A \in R \mid$ the diagonal entries of $A$ are zero $\}$ of $R$. Then $I$ is an essential ideal of $R$ whose finitely generated subrings are nilpotent since the subring is contained in $U_{2^{k}}(S)$ for some $k \geq 1$. So $R$ is ideal- $\pi$-McCoy by Lemma 1.2(3).

(3) There exists a McCoy ring that is not ideal- $\pi$-McCoy. Let $I$ be an infinite indexing set and $R_{i}$ be a ring for all $i \in I$. Let $R=\sum_{i \in I} R_{i}$ be the direct 
sum of $R_{i}$ 's. Suppose that $R_{j}$ is not ideal- $\pi$-McCoy for some $j \in I$. Then by Proposition 2.1(2), to follow, $R$ is not ideal- $\pi$-McCoy. But $R$ is McCoy by [7, Proposition 4.3] and thus $R$ is $\pi$-McCoy by [15, Proposition 1.1(2)].

McCoy rings are $\pi$-McCoy, but $\pi$-McCoy rings need not be ideal- $\pi$-McCoy by Example 1.4(3). We will see another $\pi$-McCoy ring but not ideal- $\pi$-McCoy in the following.

Example 1.5. Let $R=\operatorname{Mat}_{2}(\mathbb{Z}) \bigoplus \operatorname{Mat}_{2}\left(\mathbb{Z}_{4}\right)$. Then $\operatorname{Mat}_{2}(\mathbb{Z})$ is not $\pi$-McCoy by $\left[15\right.$, Theorem 1.4] but $\operatorname{Mat}_{2}\left(\mathbb{Z}_{4}\right)$ is non-semiprime with $N_{*}\left(\operatorname{Mat}_{2}\left(\mathbb{Z}_{4}\right)\right)=$ $\operatorname{Mat}_{2}\left(2 \mathbb{Z}_{4}\right)$; hence $R$ is $\pi$-McCoy by $\left[15\right.$, Proposition 2.7]. Note that $\operatorname{Mat}_{2}(\mathbb{Z})$ is not ideal- $\pi$-McCoy by Example $1.3(1)$, and so $R$ is not ideal- $\pi$-McCoy by Proposition 2.1(1) to follow.

For any polynomial $f(x)$ in $R[x]$, let $C_{f(x)}$ denote the set of all coefficients of $f(x)$. In [23], Rege and Chhawchharia called a ring $R$ Armendariz if whenever any polynomials $f(x), g(x) \in R[x]$ satisfy $f(x) g(x)=0$, then $a b=0$ for all $a \in C_{f(x)}$ and $b \in C_{g(x)}$. This nomenclature was used by them since it was Armendariz [4, Lemma 1] who initially showed that a reduced ring always satisfies this condition. The class of 2-primal rings and the class of Armendariz rings don't imply each other by [23, Example 3.2] and [3, Example 4.8].

Proposition 1.6. (1) Armendariz rings are ideal- $\pi-M c C o y$.

(2) 2-primal rings are ideal- $\pi-M c C o y$.

Proof. (1) Note that $R$ is Armendariz if and only if $R[x]$ is, by [1, Theorem 2 . Let $R$ be Armendariz. Suppose that $f(x) g(x) \in N(R[x])$ for nonzero polynomials $f(x), g(x)$ in $R[x]$. By [3, Corollary 5.2] and [3, Proposition 2.7], we have $a b \in N(R)$ for all $a \in C_{f(x)}$ and $b \in C_{g(x)}$ and so $f(x) b^{\prime} \in N(R)[x]=$ $N(R[x])$ for some $0 \neq b^{\prime} \in C_{g(x)}$ because $g(x) \neq 0$. Thus $R$ is ideal- $\pi$-McCoy.

(2) Note that $R[x]$ over a 2-primal ring $R$ is 2-primal by [6, Proposition 2.6], and hence $N(R[x])=N(R)[x]$. Let $R$ be a 2-primal ring. Then $R[x] / N(R)[x] \cong$ $(R / N(R))[x]$ is reduced and $N(R[x])=N(R)[x]$. Let $f(x) g(x) \in N(R[x])=$ $N(R)[x]$ for nonzero polynomials $f(x), g(x)$ in $R[x]$. Since $(R / N(R))[x]$ is reduced and so Armendariz, $a b \in N(R)$ for all $a \in C_{f(x)}$ and $b \in C_{g(x)}$. By the same argument to the proof of (1), we can show that $R$ is ideal- $\pi$-McCoy.

The converse of Proposition 1.6(1) does not hold with the help of Example 1.7 to follow. Proposition 1.6(2) implies that 2-primal rings are $\pi$-McCoy [15, Proposition 1.7], since ideal- $\pi$-McCoy rings are $\pi$-McCoy, and the converse of Proposition 1.6(2) need not hold. Let $R$ be the ring in Example 1.4(2). Then $R$ is an ideal- $\pi$-McCoy ring, but not 2-primal by [13, Example 1.2].

As a generalization of Armendariz rings, Antoine [3] called a ring $R$ nilArmendariz if $a b \in N(R)$ for all $a \in C_{f(x)}$ and $b \in C_{g(x)}$ whenever two polynomials $f(x), g(x) \in R[x]$ satisfy $f(x) g(x) \in N(R)[x]$. Nil-Armendariz rings contain both 2-primal rings and Armendariz rings, but each converse does not 
hold by [3, Example 4.8 and Example 4.9]. Hence, we raise the following question:

Question. Is a ring $R$ ideal- $\pi$-McCoy if $R$ is nil-Armendariz?

Recall that a ring $R$ is called (von Neumann) regular if for each $a \in R$ there exists $x \in R$ such that $a=a x a$. Observe that a regular $\operatorname{ring} R$ is Armendariz if and only if $R$ is nil-Armendariz if and only if $R$ is 2-primal by [18, Theorem 20].

A ring is called Abelian if every idempotent is central. Ideal- $\pi$-McCoy rings need not be Abelian as can be seen by $U_{n}(A)$ for $n \geq 2$ and any $\operatorname{ring} A$. Abelian rings are not $\pi$-McCoy by [15, Example 1.9] and so not ideal- $\pi$-McCoy. Note that a ring $R$ is Abelian regular if and only if $R$ is reduced regular [10, Theorem 3.2 . Consequently a regular ring $R$ is reduced if and only if $R$ is reversible if and only if $R$ is Abelian if and only if $R$ is 2-primal. Since the class of ideal- $\pi$-McCoy rings contains both Armendariz rings and 2-primal rings, one may conjecture that regular ideal- $\pi$-McCoy rings are Abelian (hence reduced). However the following provides a negative answer.

Example 1.7. We use the ring in [15, Example 1.10]. Let $S$ be a regular ring, $n$ be a positive integer, and $R_{n}$ be the $2^{n}$ by $2^{n}$ full matrix ring over $S$. Define a map $\sigma: R_{n} \rightarrow R_{n+1}$ by $A \mapsto\left(\begin{array}{cc}A & 0 \\ 0 & A\end{array}\right)$, then $R_{n}$ can be considered as a subring of $R_{n+1}$ via $\sigma$ (i.e., $A=\sigma(A)$ for $A \in R_{n}$ ). Notice that $D=\left\{R_{n}, \sigma_{n m}\right\}$, with $\sigma_{n m}=\sigma^{m-n}$ whenever $n \leq m$, is a direct system over $I=\{1,2, \ldots\}$. Set $R=\lim R_{n}$ be the direct limit of $D$. Then $R$ is regular since every $R_{n}$ is regular, but not reduced.

Let $f(x) g(x) \in N(R[x])$ for $0 \neq f(x), 0 \neq g(x) \in R[x]$. Then there exists $k \geq 1$ such that $f(x), g(x) \in R_{k}[x]$. Since $g(x) \neq 0$, there exists a nonzero coefficient of $g(x)$, say $\left(a_{i j}\right) \neq 0$ with $a_{p q} \neq 0$. So the ideal of $R$ generated by the coefficients of $g(x)$ contains the matrix

$$
\left(b_{s t}\right)=E_{1 p}\left(a_{i j}\right) E_{q 2^{k+1}}
$$

in $R_{k+1}$ such that the $\left(1,2^{k+1}\right)$-entry of $\left(b_{s t}\right)$ is $a_{p q}$ and other entries of $\left(b_{s t}\right)$ are all zero. Thus $\left(f(x)\left(b_{s t}\right)\right)^{2^{k+1}}=0$ and this implies that $R$ is ideal- $\pi$-McCoy.

A ring $R$ is called directly finite if $a b=1$ implies $b a=1$ for $a, b \in R$. Clearly Abelian rings are directly finite. Both strongly McCoy rings and 2-primal rings are directly finite by help of [6, Proposition 2.10] and [7, Theorem 5.2]. So one may conjecture that ideal- $\pi$-McCoy rings are directly finite. However the following erases the possibility.

Example 1.8. We use the ring and argument in [15, Example 1.8]. Let $F$ be a field and $\mathbb{V}$ be an infinite dimensional vector space over $F$ with a basis $\left\{v_{1}, v_{2}, \ldots\right\}$. Consider the endomorphism ring $R=\operatorname{End}_{F}(\mathbb{V})$ and define $f, g \in$ $R$ such that $f v_{1}=0, f v_{j}=v_{j-1}$ for $j=2,3, \ldots$ and $g v_{i}=v_{i+1}$ for $i=1,2, \ldots$ Then $f g=1$ but $g f \neq 1$. Now consider $U_{n}(R)$ for $n \geq 2$. Then $U_{n}(R)$ is 
ideal- $\pi$-McCoy by Lemma 1.2(4). Take $a=\left(a_{i j}\right)$ and $b=\left(b_{i j}\right)$ in $U_{n}(R)$ such that $a_{i i}=f$ for all $i$, elsewhere zero, and $b_{i i}=g$ for all $i$, elsewhere zero. Then $a b=1$ but $b a \neq 1$; hence $U_{n}(R)$ is not directly finite.

The following is a similar result to [16, Proposition 2.1].

Proposition 1.9. Let $R$ be an ideal- $\pi-M c$ Coy ring and suppose that $f_{1}(x), \ldots$, $f_{n}(x) \in R[x]$ are such that $f_{1}(x) \cdots f_{n}(x) \in N(R[x])$ and $f_{i}(x) \neq 0$ for all $i \in\{2, \ldots, n\}$. Then there exists a nonzero $r_{i}$ in the ideal of $R$ generated by the coefficients of $f_{i}(x)$ in $R$ such that

$$
f_{1}(x) \cdots f_{n-1}(x) r_{n}, \ldots, f_{1}(x) f_{2}(x) r_{3} \cdots r_{n}, f_{1}(x) r_{2} r_{3} \cdots r_{n} \in N(R[x])
$$

for $i=2, \ldots, n$.

Proof. Suppose that $R$ is ideal- $\pi$-McCoy. Since $\left(f_{1}(x) \cdots f_{n-1}(x)\right) f_{n}(x) \in$ $N(R[x])$, there exists a nonzero $r_{n}$ in the ideal of $R$ generated by the coefficients of $f_{n}(x)$ such that $\left(f_{1}(x) \cdots f_{n-1}(x)\right) r_{n} \in N(R[x])$. Hence we get $r_{n}\left(f_{1}(x) \cdots f_{n-1}(x)\right) \in N(R[x])$. Since $r_{n}\left(f_{1}(x) \cdots f_{n-1}(x)\right) \in N(R[x])$, there exists a nonzero $r_{n-1}$ in the ideal of $R$ generated by the coefficients of $f_{n-1}(x)$, entailing $r_{n-1} r_{n}\left(f_{1}(x) \cdots f_{n-2}(x)\right) \in N(R[x])$.

Proceeding in this manner, we finally obtain $r_{2} \cdots r_{n-1} r_{n} f_{1}(x) \in N(R[x])$ and this yields

$$
f_{1}(x) r_{2} \cdots r_{n-1} r_{n} \in N(R[x])
$$

where $r_{i}$ is a nonzero element in the ideal of $R$ generated by the coefficients of $f_{i}(x)$ for $i=2, \ldots, n$.

When we apply this proposition we should proceed our computation for each of $\left\{r_{n}, \ldots, r_{3} \cdots r_{n}, r_{2} r_{3} \cdots r_{n}\right\}$ to be nonzero.

\section{Examples of ideal- $\pi$-McCoy rings}

In this section we examine the ideal- $\pi$-McCoy property in various kinds of ordinary ring extensions.

Proposition 2.1. Let $\Gamma$ be an index set and $R_{\gamma}$ be a ring for each $\gamma \in \Gamma$.

(1) The direct product $R=\prod_{\gamma \in \Gamma} R_{\gamma}$ is ideal- $\pi-M c$ Coy if and only if $R_{\gamma}$ is ideal- $\pi-M c$ Coy for all $\gamma \in \Gamma$.

(2) The direct sum $R=\sum_{\gamma \in \Gamma} R_{\gamma}$ (possibly without identity) is ideal- $\pi$ McCoy if and only if $R_{\gamma}$ is ideal- $\pi-M c C o y$ for all $\gamma \in \Gamma$.

(3) Let $R$ be the subring of $\prod_{\gamma \in \Gamma} R_{\gamma}$ generated by $\sum_{\gamma \in \Gamma} R_{\gamma}$ and $1_{\prod_{\gamma \in \Gamma} R_{\gamma}}$. Then $R$ is ideal- $\pi-M c C o y$ if and only if $R_{\gamma}$ is ideal- $\pi-M c C o y$ for all $\gamma \in \Gamma$.

(4) Let $R$ be an ideal- $\pi-M c$ Coy ring. If $I$ is a proper right or left ideal of $R$, then $I$ is an ideal- $\pi-M c C o y$ ring (without identity).

(5) The class of ideal- $\pi-M c C o y$ rings is not closed under subrings.

(6) The class of ideal- $\pi-M c C o y$ rings is not closed under homomorphic images. 
Proof. (1) Let $f(x) g(x) \in N(R[x])$ for

$$
f(x)=\sum_{i=0}^{m}\left(a(i)_{\gamma}\right) x^{i}, 0 \neq g(x)=\sum_{j=0}^{n}\left(b(j)_{\gamma}\right) x^{j} \in R[x] .
$$

Letting $f_{\gamma}(x)=\sum_{i=0}^{m} a(i)_{\gamma} x^{i}$ and $g_{\gamma}(x)=\sum_{j=0}^{n} b(j)_{\gamma} x^{j}$, we can write $f(x)=$ $\left(f_{\gamma}(x)\right)$ and $g(x)=\left(g_{\gamma}(x)\right)$. From $f(x) g(x) \in N(R[x])$, we get $f_{\gamma}(x) g_{\gamma}(x) \in$ $N\left(R_{\gamma}[x]\right)$ for all $\gamma \in \Gamma$. Suppose that each $\operatorname{ring} R_{\gamma}$ is ideal- $\pi$-McCoy. Since $g(x) \neq 0$ there exists some index $k \in \Gamma$ such that $g_{k}(x) \neq 0$. Then since $R_{k}$ is ideal- $\pi$-McCoy, there exists some nonzero $r_{k}$ in the ideal of $R_{k}$ generated by the coefficients of $g_{k}(x)$ such that $f_{k}(x) r_{k} \in N\left(R_{k}[x]\right)$. Say $r_{k}=\sum_{\lambda \in \Lambda} s_{\lambda} d_{\lambda} t_{\lambda}$ where $s_{\lambda}, t_{\lambda} \in R_{k}, d_{\lambda} \in\left\{b \mid b\right.$ is a coefficient of $\left.g_{k}(x)\right\}$, and $\Lambda$ is finite. Let $r=\left(r_{\gamma}\right) \in R$ be the sequence with $r_{\gamma}=r_{k}$ for $\gamma=k$ and $r_{\gamma}=0$ for $\gamma \neq k$; and $\left(u(\lambda)_{\gamma}\right)$ be a coefficient of $g(x)$ for $\lambda \in \Lambda$ such that $u(\lambda)_{\gamma}=d_{\lambda}$ for $\gamma=k$. Then $r=\sum_{\lambda \in \Lambda}\left(s(\lambda)_{\gamma}\right)\left(u(\lambda)_{\gamma}\right)_{\lambda}\left(t(\lambda)_{\gamma}\right)$ where $s(\lambda)_{\gamma}=s_{\lambda}, t(\lambda)_{\gamma}=t_{\lambda}$ for $\gamma=k$ and $s(\lambda)_{\gamma}=0, t(\lambda)_{\gamma}=0$ for $\gamma \neq k$. This yields $f(x) r \in N(R[x])$, and so $R$ is ideal- $\pi$-McCoy.

Conversely, let $R$ be ideal- $\pi$-McCoy, and assume on the contrary that $R_{\gamma_{0}}$ is not ideal- $\pi$-McCoy for some $\gamma_{0} \in \Gamma$. Then there exist $f_{\gamma_{0}}(x), 0 \neq g_{\gamma_{0}}(x)$ in $R_{\gamma_{0}}[x]$ such that $f_{\gamma_{0}}(x) g_{\gamma_{0}}(x) \in N\left(R_{\gamma_{0}}[x]\right)$ but $f_{\gamma_{0}}(x) r_{\gamma_{0}} \notin N\left(R_{\gamma_{0}}[x]\right)$ for all $0 \neq r_{\gamma_{0}}$ in the ideal of $R_{\gamma_{0}}$ generated by the coefficients of $g_{\gamma_{0}}(x)$. Taking $f(x)=\left(f_{\gamma}(x)\right), g(x)=\left(g_{\gamma}(x)\right)$ such that $f(x)$ and $g(x)$ are the sequences in $R[x]$ such that $f_{\gamma}(x)=f_{\gamma_{0}}(x)$ for $\gamma=\gamma_{0}, f_{\gamma}(x)=0$ for $\gamma \neq \gamma_{0}$, and $g_{\gamma}(x)=g_{\gamma_{0}}(x)$ for $\gamma=\gamma_{0}, g_{\gamma}(x)=0$ for $\gamma \neq \gamma_{0}$. Then $f(x) g(x) \in N(R[x])$ from $f_{\gamma_{0}}(x) g_{\gamma_{0}}(x) \in N\left(R_{\gamma_{0}}[x]\right)$. But since $R$ is ideal- $\pi$-McCoy, there exists a nonzero $s=\left(s_{\gamma}\right)$ in the ideal of $R$ generated by the coefficients of $g(x)$ such that $f(x) s \in N(R[x])$. Note that $s_{\gamma} \neq 0$ for $\gamma=\gamma_{0}$ and $s_{\gamma}=0$ for $\gamma \neq \gamma_{0}$ and that $s_{\gamma_{0}}$ is in the ideal of $R_{\gamma_{0}}$ generated by the coefficients of $g_{\gamma_{0}}(x)$. This yields $f_{\gamma_{0}}(x) s_{\gamma_{0}} \in N\left(R_{\gamma_{0}}[x]\right)$, a contradiction.

The proofs of (2) and (3) are much the same as (1).

(4) Let $f(x) g(x) \in N(I[x])$ for polynomials $f(x), 0 \neq g(x)$ in $I[x]$. Since $f(x) g(x) \in N(I[x]) \subseteq N(R[x])$ and $R$ is ideal- $\pi$-McCoy, $f(x) r \in N(R[x])$ for some $0 \neq r$ in the ideal of $R$ generated by the coefficients of $g(x)$. Since $I$ is an ideal of $R, r \in I$ and $f(x) r \in N(I[x])$. So $I$ is an ideal- $\pi$-McCoy ring without identity.

(5) Let $R=\operatorname{Mat}_{n}(S)$ for a reduced $\operatorname{ring} S$ and $n \geq 2$. Then $R$ is not ideal- $\pi$-McCoy by Example 1.3(1). But $U_{2}(S)$ is ideal- $\pi$-McCoy by Lemma $1.2(4)$.

(6) Let $R$ be the ring of quaternions with integer coefficients. Then $R$ is a domain and clearly ideal- $\pi$-McCoy. However for any odd prime integer $q$, the $\operatorname{ring} R / q R$ is isomorphic to $\operatorname{Mat}_{2}\left(\mathbb{Z}_{q}\right)$ by the argument in [11, Exercise $\left.2 \mathrm{~A}\right]$. Thus $R / q R$ is not ideal- $\pi$-McCoy by Example $1.3(1)$.

The construction in Example 1.7 also provides an ideal- $\pi$-McCoy ring which has a non-ideal- $\pi$-McCoy subring. Let $S$ be a division ring in Example 1.7. 
Then every $R_{n}=\operatorname{Mat}_{2^{n}}(S)$ is not ideal- $\pi$-McCoy by Example 1.3(1). But $R$ is ideal- $\pi$-McCoy by the computation in Example 1.7. Note that every $R_{n}$ is a subring of $R$.

We find a kind of subring that inherits the ideal- $\pi$-McCoy property against Proposition 2.1(5).

Corollary 2.2. Let $e$ be a central idempotent of a ring $R$. Then $R$ is ideal- $\pi$ $M c C o y$ if and only if $e R$ and $(1-e) R$ are both ideal- $\pi-M c C o y$.

Proof. The proof is obtained from Proposition 2.1(1) since $R=e R \oplus(1-$ e) $R$.

Concerning the preceding corollary, we write an actual computation to show the sufficiency. Suppose that $e R$ and $(1-e) R$ are both ideal- $\pi$-McCoy. Let $f(x)=\sum_{i=0}^{m} a_{i} x^{i}$ and $g(x)=\sum_{j=0}^{n} b_{j} x^{j} \in R[x]$ be such that $f(x) g(x) \in$ $N(R[x])$ and $g(x) \neq 0$. Next set $f_{1}(x)=e f(x), f_{2}(x)=(1-e) f(x)$ and $g_{1}(x)=e g(x), g_{2}(x)=(1-e) g(x)$. Then $f_{1}(x) g_{1}(x)=e f(x) g(x) \in N(R[x])$ and $f_{2}(x) g_{2}(x)=(1-e) f(x) g(x) \in N(R[x])$. Since $g(x) \neq 0$, eg $(x) \neq 0$ or $(1-e) g(x) \neq 0$.

Assume $g_{1}(x) \neq 0$ and $g_{2}(x) \neq 0$. Since $e R$ (resp. $(1-e) R$ ) is ideal- $\pi$-McCoy, there exists $r_{1} \neq 0$ (resp. $r_{2} \neq 0$ ) in the ideal of $e R$ (resp. $(1-e) R$ ) generated by the coefficients of $g_{1}(x)$ (resp. $g_{2}(x)$ ) such that $f_{1}(x) r_{1} \in N(e R[x]$ ) (resp. $\left.f_{2}(x) r_{2} \in N((1-e) R[x])\right)$. Let $r=r_{1}+r_{2}$. Then $r \neq 0$ since $e R \cap(1-e) R=0$, and $r$ is contained in the ideal of $R$ generated by the coefficients of $g(x)$. Moreover we have

$$
f(x) r=\left(f_{1}(x)+f_{2}(x)\right)\left(r_{1}+r_{2}\right)=f_{1}(x) r_{1}+f_{2}(x) r_{2} \in N(R[x]) .
$$

The computations of the cases of $\left(g_{1}(x) \neq 0, g_{2}(x)=0\right)$ and $\left(g_{1}(x)=0, g_{2}(x) \neq\right.$ $0)$ are similar. These imply that $R$ is ideal- $\pi$-McCoy.

Theorem 2.3. The class of ideal- $\pi-M c$ Coy rings is closed under direct limits with injective maps.

Proof. Let $D=\left\{R_{i}, \alpha_{i j}\right\}$ be a direct system of ideal- $\pi$-McCoy rings $R_{i}$ for $i \in I$ and ring homomorphisms $\alpha_{i j}: R_{i} \rightarrow R_{j}$ for each $i \leq j$ satisfying $\alpha_{i j}(1)=1$, where $I$ is a directed partially ordered set. Set $R=\lim R_{i}$ be the direct limit of $D$ with $\iota_{i}: R_{i} \rightarrow R$ and $\iota_{j} \alpha_{i j}=\iota_{i}$, where every $\iota_{i}$ is injective. We will show that $R$ is an ideal- $\pi$-McCoy ring. Take $a, b \in R$. Then $a=\iota_{i}\left(a_{i}\right), b=\iota_{j}\left(b_{j}\right)$ for some $i, j \in I$ and there is $k \in I$ such that $i \leq k, j \leq k$. Define

$$
a+b=\iota_{k}\left(\alpha_{i k}\left(a_{i}\right)+\alpha_{j k}\left(b_{j}\right)\right) \text { and } a b=\iota_{k}\left(\alpha_{i k}\left(a_{i}\right) \alpha_{j k}\left(b_{j}\right)\right),
$$

where $\alpha_{i k}\left(a_{i}\right)$ and $\alpha_{j k}\left(b_{j}\right)$ are in $R_{k}$. Then $R$ forms a ring with $0=\iota_{i}(0)$ and $1=\iota_{i}(1)$.

Now let $f(x), g(x) \in R[x]$ be nonzero polynomials such that $f(x) g(x) \in$ $N(R[x])$. There is $k \in I$ such that $f(x), g(x) \in R_{k}[x]$ via $\iota_{i}$ 's and $\alpha_{i j}$ 's; hence we get $f(x) g(x) \in N\left(R_{k}[x]\right)$. Since $R_{k}$ is ideal- $\pi$-McCoy, there exists $0 \neq c_{k}$ in the ideal of $R_{k}$ generated by the coefficients of $g(x)$ such that $f(x) c_{k} \in N\left(R_{k}[x]\right)$. 
Put $c=\iota_{k}\left(c_{k}\right)$. Then $f(x) c \in N(R[x])$ with a nonzero $c$ in the ideal of $R$ generated by the coefficients of $g(x)$, entailing $R$ being ideal- $\pi$-McCoy.

Write $R$ and $R_{n}$ as in Example 1.7. Then the direct limit $R$ of $R_{n}$ 's is ideal- $\pi$-McCoy, but $R_{n}$ need not ideal- $\pi$-McCoy with the help of [15, Theorem $1.4]$.

The class of ideal- $\pi$-McCoy rings is not closed under subrings by Proposition 2.1(5). This is comparable with the following.

Proposition 2.4. Let $R$ be a ring. If $R[x]$ is ideal- $\pi-M c$ Coy over a ring $R$, then so is $R$.

Proof. Suppose that $S[t]$ is the polynomial ring with an indeterminate $t$ over $S=R[x]$. We apply the proof of [15, Proposition 2.3]. Let $S$ be ideal- $\pi$-McCoy and suppose that $f(x) g(x) \in N(R[x])$ for nonzero polynomials $f(x), g(x)$ in $R[x]$. This can be converted to that $f(t) g(t) \in N(S[t])$ for nonzero polynomials $f(t), g(t)$ in $S[t]$. Since $S$ is ideal- $\pi$-McCoy, $f(t) h(x) \in N(S[t])$ for some $0 \neq$ $h(x)$ in the ideal of $R[x]$ generated by the coefficients of $g(t)$, say $(f(t) h(x))^{k}=$ 0 . Note that $f(t) \in R[t]$. Here letting $h(x)=\sum_{i=0}^{n} a_{i} x^{i}$ (we can set $a_{0} \neq 0$, dividing by $x$ if necessary), we get $f(t) a_{0} \in N(R[t])$ from $0=(f(t) h(x))^{k}=$ $\left(f(t) a_{0}\right)^{k}+h_{1} x+\cdots+h_{n k} x^{n k}$ with $h_{1}, \ldots, h_{n k} \in R[t]$. This implies that $f(x) a_{0} \in N(R[x])$ and $0 \neq a_{0}$ in the ideal of $R$ generated by the coefficients of $g(x)$, showing that $R$ is ideal- $\pi$-McCoy.

In fact, we do not know of any example of an ideal- $\pi$-McCoy ring whose polynomial ring is not ideal- $\pi$-McCoy.

Question. Does a ring $R$ being ideal- $\pi$-McCoy imply $R[x]$ being ideal- $\pi$ McCoy?

Recall that a regular element means a neither left nor right zero-divisor. A $\operatorname{ring} R$ is called right Ore if given $a, b \in R$ with $b$ regular there exist $a_{1}, b_{1} \in R$ with $b_{1}$ regular such that $a b_{1}=b a_{1}$. It is well-known that $R$ is a right Ore ring if and only if the classical right quotient ring of $R$ exists.

Theorem 2.5. Let $R$ be a right Ore ring with its classical right quotient ring $Q$. Let $R$ be an ideal- $\pi-M c C o y$ ring such that non-regular polynomials in $R[x]$ are nilpotent. Then $Q$ is ideal- $\pi-M c C o y$.

Proof. We will use [20, Proposition 2.1.16] freely, and apply the proof of [15, Theorem 2.1]. Denote the set of all regular elements in $R$ by $C(R)$. Suppose $F(x) G(x) \in N(Q[x])$ for $0 \neq F(x), 0 \neq G(x) \in Q[x]$. Say that $(F(x) G(x))^{k}=$ 0 and $(F(x) G(x))^{k-1} \neq 0$ for some $k \geq 1$. We can write

$$
F(x)=\sum_{i=0}^{m} a_{i} u^{-1} x^{i}=\left(\sum_{i=0}^{m} a_{i} x^{i}\right) u^{-1} \text { and } G(x)=\sum_{j=0}^{n} b_{j} v^{-1} x^{j}=\left(\sum_{j=0}^{n} b_{j} x^{j}\right) v^{-1}
$$

for some $a_{i}$ 's, $b_{j}$ 's in $R$ and $u, v \in C(R)$. Since $R$ is right Ore, there exist $u_{1}, v_{1} \in C(R)$ for all $i$ 's and $j$ 's such that $u^{-1} b_{j}=b_{j}^{\prime} u_{1}^{-1}$ and $v^{-1} a_{i}=a_{i}^{\prime} v_{1}^{-1}$ 
for some $a_{i}^{\prime}, b_{j}^{\prime} \in R$. Next set

$$
\begin{aligned}
f(x) & =\sum_{i=0}^{m} a_{i} x^{i}, f_{1}(x)=\sum_{i=0}^{m} a_{i}^{\prime} x^{i}, \\
g(x) & =\sum_{j=0}^{n} b_{j} x^{j}, g_{1}(x)=\sum_{j=0}^{n} b_{j}^{\prime} x^{j} \text { and } \\
v_{2} & =u v_{1}, u_{2}=v u_{1} .
\end{aligned}
$$

Then we have

$$
\begin{aligned}
u^{-1} g(x) & =g_{1}(x) u_{1}^{-1}, v^{-1} f(x)=f_{1}(x) v_{1}^{-1}, \\
F(x) G(x) & =f(x) g_{1}(x) u_{2}^{-1} \text { and } \\
G(x) F(x) & =g(x) f_{1}(x) v_{2}^{-1},
\end{aligned}
$$

noting that $f(x) \neq 0, g(x) \neq 0, f_{1}(x) \neq 0$, and $g_{1}(x) \neq 0$. Let $I$ and $J$ be the ideals of $Q$ generated by the coefficients of $F(x)$ and $G(x)$, respectively. Since

$$
a_{i}=a_{i} u^{-1} u, u^{-1} b_{j} u_{1}=b_{j}^{\prime}, b_{j}=b_{j} v^{-1} v, v^{-1} a_{i} v_{1}=a_{i}^{\prime},
$$

we have $f(x), f_{1}(x) \in I[x]$ and $g(x), g_{1}(x) \in J[x]$. Note that $\sum_{i=0}^{m} R a_{i} R \subseteq I$, $\sum_{i=0}^{m} R a_{i}^{\prime} R \subseteq I, \sum_{j=0}^{n} R b_{j} R \subseteq J, \sum_{j=0}^{n} R b_{j}^{\prime} R \subseteq J, \sum_{i=0}^{m} R a_{i}^{\prime} R \subseteq R$, and $\sum_{j=0}^{n} R b_{j}^{\prime} R \subseteq R$. We will freely use this fact in the following computation. Set $I_{0}=\sum_{i=0}^{m} R a_{i}^{\prime} R$ and $J_{0}=\sum_{j=0}^{n} R b_{j}^{\prime} R$.

Case 1. $F(x) G(x)=0$ and $G(x) F(x)=0$

Consider $F(x) G(x)=0$. Then $f(x) g_{1}(x) u_{2}^{-1}=0$ and so $f(x) g_{1}(x)=0$. Since $R$ is ideal- $\pi$-McCoy and $f(x), g_{1}(x) \in R[x]$, there exists $0 \neq \alpha \in J_{0}$ such that $f(x) \alpha \in N(R[x]) \subseteq N(Q[x])$. This yields

$$
F(x) u \alpha=f(x) u^{-1} u \alpha=f(x) \alpha \in N(Q[x]),
$$

where $0 \neq u \alpha \in J$.

Consider $G(x) F(x)=0$. Then $g(x) f_{1}(x) v_{2}^{-1}=0$ and so $g(x) f_{1}(x)=0$. Since $R$ is ideal- $\pi$-McCoy and $g(x), f_{1}(x) \in R[x]$, there exists $0 \neq \beta \in I_{0}$ such that $g(x) \beta \in N(R[x]) \subseteq N(Q[x])$ by the similar argument to above. This yields

$$
G(x) v \beta=g(x) v^{-1} v \beta=g(x) \beta \in N(Q[x]),
$$

where $0 \neq v \beta \in I$. Note $v \beta G(x) \in N(Q[x])$.

Case 2. $F(x) G(x)=0$ and $G(x) F(x) \neq 0$

Note that $G(x) F(x) G(x)=0$ and $F(x) G(x) F(x)=0$. Letting $H(x)=$ $G(x) F(x)$, we have $H(x) G(x)=0$. Note $0 \neq H(x) \in(I \cap J)[x]$. Say $H(x)=$ $\sum_{s=0}^{\ell} c_{s} w^{-1} x^{s}=\left(\sum_{s=0}^{\ell} c_{s} x^{s}\right) w^{-1}$ with $c_{s}$ 's in $R$ and $w \in C(R)$.

Consider $H(x) G(x)=0$. Since $R$ is right Ore, there exists $w_{1} \in C(R)$ for all $\ell^{\prime}$ 's such that $w^{-1} b_{j}=d_{j} w_{1}^{-1}$ for some $d_{j} \in R$. Letting $h(x)=$ 
$\sum_{s=0}^{\ell} c_{s} x^{s}$ and $g_{2}(x)=\sum_{j=0}^{n} d_{j} x^{j}$, we have $H(x) G(x)=h(x) g_{2}(x)\left(v w_{1}\right)^{-1}$ and so $h(x) g_{2}(x)=0$. Note

$$
w^{-1} G(x)=w^{-1} g(x) v^{-1}=g_{2}(x) w_{1}^{-1} v^{-1}=g_{2}(x)\left(v w_{1}\right)^{-1} .
$$

Since $R$ is ideal- $\pi$-McCoy and $h(x), g_{2}(x) \in R[x]$, there exists $0 \neq \gamma \in \sum_{s=0}^{\ell} R c_{s} R$ such that $\gamma g_{2}(x) \in N(R[x]) \subseteq N(Q[x])$. Note that $\gamma \in I \cap J, g_{2}(x) \gamma \in N(Q[x])$ and

$$
w^{-1} G(x) v w_{1} \gamma=g_{2}(x)\left(v w_{1}\right)^{-1} v w_{1} \gamma=g_{2}(x) \gamma \in N(Q[x]) .
$$

This yields $G(x) v w_{1} \gamma w^{-1} \in N(Q[x])$ with $0 \neq v w_{1} \gamma w^{-1} \in I \cap J$.

Next since $F(x) G(x)=0$, the same computation as in Case 1 is applicable to find nonzero $q \in J$ such that $F(x) q \in N(Q[x])$.

Case 3. $F(x) G(x) \neq 0$ and $G(x) F(x)=0$

Note that $F(x) G(x) F(x)=0$ and $G(x) F(x) G(x)=0$. Letting $K(x)=$ $F(x) G(x)$, we have $K(x) F(x)=0$ with $0 \neq K(x) \in(I \cap J)[x]$. Note $0 \neq$ $K(x) \in(I \cap J)[x]$. Say $K(x)=\sum_{s=0}^{t} e_{s} z^{-1} x^{s}=\left(\sum_{s=0}^{t} e_{s} x^{s}\right) z^{-1}$ with $e_{s}$ 's in $R$ and $z \in C(R)$.

Since $R$ is right Ore, there exists $z_{1} \in C(R)$ for all $i$ 's such that $z^{-1} a_{i}=y_{i} z_{1}^{-1}$ for some $y_{i} \in R$. Let $k(x)=\sum_{s=0}^{t} e_{s} x^{s}$ and $f_{2}(x)=\sum_{i=0}^{m} y_{i} x^{i}$. Then $k(x) \neq 0$ and we have $K(x) F(x)=k(x) f_{2}(x)\left(u z_{1}\right)^{-1}$, entailing $k(x) f_{2}(x)=0$. Note

$$
z^{-1} F(x)=z^{-1} f(x) u^{-1}=f_{2}(x) z_{1}^{-1} u^{-1}=f_{2}(x)\left(u z_{1}\right)^{-1} .
$$

Since $R$ is ideal- $\pi$-McCoy and $k(x), f_{2}(x) \in R[x]$, there exists $0 \neq \delta \in \sum_{s=0}^{t} R e_{s} R$ such that $\delta f_{2}(x) \in N(R[x]) \subseteq N(Q[x])$. Note that $\delta \in I \cap J, f_{2}(x) \delta \in N(Q[x])$ and

$$
z^{-1} F(x) u z_{1} \delta=f_{2}(x)\left(u z_{1}\right)^{-1} u z_{1} \delta=f_{2}(x) \delta \in N(Q[x]) .
$$

This yields $F(x) u z_{1} \delta z^{-1} \in N(Q[x])$ with $0 \neq u z_{1} \delta z^{-1} \in I \cap J$.

Next since $G(x) F(x)=0$, the same computation as in Case 1 is applicable to find nonzero $p \in I$ such that $p G(x) \in N(Q[x])$.

Case 4. $F(x) G(x) \neq 0$ and $G(x) F(x) \neq 0$

Suppose $F(x) G(x) \neq 0$ and $G(x) F(x) \neq 0$. Then there exists $k \geq 2$ such that $(F(x) G(x))^{k}=0$ and $(F(x) G(x))^{k-1} \neq 0$.

Since $R$ is right Ore, $u_{2}^{-1}(F(x) G(x))^{k-1}=A(x) u_{3}^{-1}$ for some $A(x) \in R[x]$ and some $u_{3} \in C(R)$. Here $A(x)$ is also nonzero because both $(F(x) G(x))^{k-1}$ is nonzero. Note that $A(x) \in(I \cap J)[x]$ and

$$
f(x) g_{1}(x) A(x)=f(x) g_{1}(x) u_{2}^{-1}(F(x) G(x))^{k-1} u_{3}=(F(x) G(x))^{k} u_{3}=0 .
$$

Since $R$ is ideal- $\pi$-McCoy, there exists nonzero $\beta$ in the ideal of $R$ generated by the coefficients of $A(x)$ such that $f(x) g_{1}(x) \beta \in N(R[x])$. Note $\beta \in I \cap J$. Moreover $g_{1}(x) \beta$ is nilpotent by hypothesis. Then, from

$$
g_{1}(x) \beta=g_{1}(x) u_{2}^{-1} u_{2} \beta=u^{-1} g(x) v^{-1} u_{2} \beta=u^{-1} G(x) u_{2} \beta,
$$

we have $G(x) u_{2} \beta u^{-1} \in N(Q[x])$ with $0 \neq u_{2} \beta u^{-1} \in I \cap J$. 
By Cases 1, 2, 3 and 4, we have that

$$
\beta^{\prime} G(x) \in N(Q[x]) \text { for some } 0 \neq \beta^{\prime} \in I .
$$

Therefore $Q$ is ideal- $\pi$-McCoy by help of Proposition 1.1(1).

The following has a similar structure to the case of classical quotient rings.

Proposition 2.6. Let $R$ be a ring and $\Delta$ be a multiplicatively closed subset of $R$ consisting of central regular elements. Then

(1) $R$ is ideal- $\pi-M c C o y$ if and only if $\Delta^{-1} R$ is.

(2) $R$ is strongly right $M c$ Coy if and only if $\Delta^{-1} R$ is.

Proof. The necessity follows from the proof of Theorem 2.5. Let $S=\Delta^{-1} R$ and put $f(x)=\sum_{i=0}^{m} a_{i} x^{i}$ and $g(x)=\sum_{j=0}^{n} b_{j} x^{j}$ be nonzero polynomials in $R[x]$.

(1) Suppose that $S$ is ideal- $\pi$-McCoy and $f(x) g(x) \in N(R[x])$. Since $S$ is ideal- $\pi$-McCoy, $f(x)\left(c w^{-1}\right) \in N(S[x])$ for some nonzero $c w^{-1}$ in the ideal of $S$ generated by the coefficients of $g(x)$. Note that $c \neq 0$ and $c$ is also contained in the ideal of $S$ generated by the coefficients of $g(x)$. But since $w$ is central, we get $f(x) c \in N(R[x])$, concluding that $R$ is ideal- $\pi$-McCoy.

(2) is similar to the proof of (1).

Corollary 2.7. Let $R$ be a ring. Then $R[x]$ is ideal- $\pi-M c C o y$ (resp. strongly right $M c C o y)$ if and only if $R\left[x ; x^{-1}\right]$ is.

Proof. Note that $\Delta=\left\{1, x, x^{2}, \ldots\right\}$ is a multiplicatively closed subset of $R[x]$ consisting of central regular elements. So Proposition 2.6 gives the proof since $R\left[x ; x^{-1}\right]=\Delta^{-1} R[x]$.

Let $R$ be an algebra (with or without identity) over a commutative ring $S$. The Dorroh extension of $R$ by $S$ is the Abelian group $R \oplus S$ with multiplication given by $\left(r_{1}, s_{1}\right)\left(r_{2}, s_{2}\right)=\left(r_{1} r_{2}+s_{1} r_{2}+s_{2} r_{1}, s_{1} s_{2}\right)$ for $r_{i} \in R$ and $s_{i} \in S$.

Theorem 2.8. Let $R$ be an algebra over a commutative domain $S$, and $D$ be the Dorroh extension of $R$ by $S$. Then

(1) $R$ is ideal- $\pi-M c C o y$ if and only if $D$ is.

(2) $R$ is strongly right $M c$ Coy if and only if $D$ is.

(3) $R$ is $\pi$-McCoy if and only if $D$ is.

Proof. Note that $s \in S$ is identified with $s 1 \in R$ and so $R=\{r+s \mid(r, s) \in D\}$ and $S$ is considered as a subring of $R$. Let $F(x)=\left(f_{1}(x), f_{2}(x)\right)$ and $G(x)=$ $\left(g_{1}(x), g_{2}(x)\right)$ be any nonzero polynomials in $D[x]$ where $f_{1}(x)=\sum_{i=1}^{m} a_{i} x^{i}$, $g_{1}(x)=\sum_{j=1}^{n} b_{j} x^{j} \in R[x]$ and $f_{2}(x)=\sum_{i=1}^{m} s_{i} x^{i}, g_{2}(x)=\sum_{j=1}^{n} t_{j} x^{j} \in S[x]$.

(1) Suppose that $R$ is ideal- $\pi$-McCoy. Let $(F(x) G(x))^{k}=0$ and

$$
(F(x) G(x))^{k-1} \neq 0
$$

for some $k \geq 1$. Then $\left(f_{2}(x) g_{2}(x)\right)^{k}=0$ and so we have $f_{2}(x)=0$ or $g_{2}(x)=0$ since $S[x]$ is a domain. 
(i) If $f_{2}(x)=0$, then we have $f_{1}(x)\left(g_{1}(x)+g_{2}(x)\right) \in N(R[x])$ from $0=$ $(F(x) G(x))^{k}=\left(f_{1}(x)\left(g_{1}(x)+g_{2}(x)\right), 0\right)^{k}$. Since $R$ is ideal- $\pi$-McCoy, there exists a nonzero $r=\sum_{u, v} r_{u_{v}}\left(b_{u}+t_{u}\right) r_{u_{v}}^{\prime} \in \sum_{j=1}^{n} R\left(b_{j}+t_{j}\right) R$ such that $f_{1}(x) r \in$ $N(R[x])$. Then

$$
F(x)(r, 0) \in N(D[x])
$$

where $0 \neq(r, 0)=\sum_{u, v}\left(r_{u_{v}}, 0\right)\left(b_{u}, t_{u}\right)\left(r_{u_{v}}^{\prime}, 0\right) \in \sum_{j=1}^{n} D\left(b_{j}, t_{j}\right) D$, entailing that $D$ is ideal- $\pi$-McCoy.

(ii) Let $g_{2}(x)=0$. By the similar argument to (i), we have $\left(f_{1}(x)+\right.$ $\left.f_{2}(x)\right) g_{1}(x) \in N(R[x])$. Since $R$ is ideal- $\pi$-McCoy, there exists a nonzero $r=\sum_{u, v} r_{u_{v}} b_{u} r_{u_{v}}^{\prime} \in \sum_{j=0}^{n} R b_{j} R$ such that $\left(f_{1}(x)+f_{2}(x)\right) r \in N(R[x])$. Then $F(x)(r, 0) \in N(D[x])$, where $0 \neq(r, 0)=\sum_{u, v}\left(r_{u_{v}}, 0\right)\left(b_{u}, 0\right)\left(r_{u_{v}}^{\prime}, 0\right) \in$ $\sum_{j=0}^{n} D\left(b_{j}, 0\right) D$, showing that $D$ is ideal- $\pi$-McCoy.

By (i) and (ii), $D$ is ideal- $\pi$-McCoy.

Conversely, suppose that $D$ is ideal- $\pi$-McCoy. Let $f(x)=\sum_{i=0}^{m} a_{i} x^{i}$ and $g(x)=\sum_{j=0}^{n} b_{j} x^{j}$ in $R[x]$ be nonzero polynomials with $f(x) g(x) \in N(R[x])$, say $(f(x) g(x))^{k}=0$ but $(f(x) g(x))^{k-1} \neq 0$ for some $k \geq 1$. We take $F(x)=$ $(f(x), 0)$ and $G(x)=(g(x), 0)$ in $D[x]$. Then $(F(x) G(x))^{k}=0$ and so $F(x) G(x)$ $\in N(D[x])$. Since $D$ is ideal- $\pi$-McCoy, there exists a nonzero $(r, s)=\sum_{u, v}\left(r_{u_{v}}\right.$, $\left.s_{u_{v}}\right)\left(b_{u}, 0\right)\left(r_{u_{v}}^{\prime}, s_{u_{v}}^{\prime}\right) \in \sum_{j=0}^{n} D\left(b_{j}, 0\right) D$ with $F(x)(r, s) \in N(D[x])$. Since $(r, s)$ $=\left(\sum_{u, v}\left(r_{u_{v}}+s_{u_{v}}\right) b_{u}\left(r_{u_{v}}^{\prime}+s_{u_{v}}^{\prime}\right), 0\right)$, we have $0 \neq r=\sum_{u, v}\left(r_{u_{v}}+s_{u_{v}}\right) b_{u}\left(r_{u_{v}}^{\prime}+\right.$ $\left.s_{u_{v}}^{\prime}\right) \in \sum_{j=0}^{n} R b_{j} R$ and $f(x) r=0$. Therefore $R$ is ideal- $\pi$-McCoy.

(2) and (3) are similar to the proof of (1).

Finally, we characterize the class of noncommutative ideal- $\pi$-McCoy rings of minimal order. $|-|$ means the cardinality.

Proposition 2.9. Let $R$ be an ideal- $\pi-M c$ Coy ring. If $R$ is a noncommutative ideal- $\pi-M c$ Coy ring of minimal order, then $R$ is of order 8 and is isomorphic to $U_{2}\left(\mathbb{Z}_{2}\right)$.

Proof. Let $R$ be a noncommutative ideal- $\pi$-McCoy of minimal order. Then $|R| \geq 2^{3}$ by $\left[9\right.$, Theorem]. If $|R|=2^{3}$, then $R$ is isomorphic to $U_{2}\left(\mathbb{Z}_{2}\right)$ by $[9$, Proposition]. But $U_{2}\left(\mathbb{Z}_{2}\right)$ is an ideal- $\pi$-McCoy ring by Lemma 1.2(4). This yields that $R$ is of order 8 and is isomorphic to $U_{2}\left(\mathbb{Z}_{2}\right)$.

Acknowledgments. The authors thank the referee for very careful reading of the manuscript and many valuable suggestions that improved the paper by much. This work was supported by a 2-Year Research Grant of Pusan National University.

\section{References}

[1] D. D. Anderson and V. Camillo, Armendariz rings and gaussian rings, Comm. Algebra 26 (1998), no. 7, 2265-2272.

[2] - Semigroups and rings whose zero products commute, Comm. Algebra 27 (1999), no. $6,2847-2852$. 
[3] R. Antoine, Nilpotent elements and Armendariz rings, J. Algebra 319 (2008), no. 8, 3128-3140.

[4] E. P. Armendariz, A note on extensions of Baer and P.P.-rings, J. Aust. Math. Soc. 18 (1974), 470-473.

[5] H. E. Bell, Near-rings in which each element is a power of itself, Bull. Aust. Math. Soc. 2 (1970), 363-368.

[6] G. F. Birkenmeier, H. E. Heatherly, and E. K. Lee, Completely prime ideals and associated radicals, Ring theory (Granville, OH, 1992), 102-129, World Sci. Publ., River Edge, NJ, 1993.

[7] V. Camillo and P. P. Nielsen, McCoy rings and zero-divisors, J. Pure Appl. Algebra 212 (2008), no. 3, 599-615.

[8] P. M. Cohn, Reversible rings, Bull. London Math. Soc. 31 (1999), no. 6, 641-648.

[9] K. E. Eldridge, Orders for finite noncommutative rings with unity, Amer. Math. Monthly 73 (1968), 512-514.

[10] K. R. Goodearl, Von Neumann Regular Rings, Pitman, London-San Francisco-Melbourne, 1979.

[11] K. R. Goodearl and R. B. Warfield, Jr., An Introduction to Noncommutative Noetherian Rings, Cambridge University Press, 1989.

[12] C. Y. Hong, Y. C. Jeon, N. K. Kim, and Y. Lee, The McCoy condition on noncommutative rings, Comm. Algebra 39 (2011), no. 5, 1809-1825.

[13] S. U. Hwang, Y. C. Jeon, and Y. Lee, Structure and topological conditions of NI rings, J. Algebra 302 (2006), no. 1, 186-199.

[14] Y. C. Jeon, H. K. Kim, Y. Lee, and J. S. Yoon, On weak Armendariz rings, Bull. Korean Math. Soc. 46 (2009), no. 1, 135-146.

[15] Y. C. Jeon, H. K. Kim, N. K. Kim, T. K. Kwak, Y. Lee, and D. E. Yeo, On a generalization of the McCoy condition, J. Korean Math. Soc. 47 (2010), no. 6, 1269-1282.

[16] N. K. Kim and Y. Lee, On a ring property unifying reversible and right duo rings, J. Korean Math. Soc. (to appear).

[17] _ Extensions of reversible rings, J. Pure Appl. Algebra 185 (2003), no. 1-3, 207223.

[18] T. K. Kwak and Y. Lee, Rings over which coefficients of nilpotent polynomials are nilpotent, Internat. J. Algebra Comput. 21 (2011), no. 5, 745-762.

[19] Z. Lei, J. Chen, and Z. Ying, A question on McCoy rings, Bull. Aust. Math. Soc. 76 (2007), no. 1, 137-141.

[20] J. C. McConnell and J. C. Robson, Noncommutative Noetherian Rings, John Wiley \& Sons Ltd., 1987.

[21] N. H. McCoy, Remarks on divisors of zero, Amer. Math. Monthly 49 (1942), 286-295.

[22] P. P. Nielsen, Semi-commutativity and the McCoy condition, J. Algebra 298 (2006), no. $1,134-141$.

[23] M. B. Rege and S. Chhawchharia, Armendariz rings, Proc. Japan Acad. Ser. A Math. Sci. 73 (1997), no. 1, 14-17.

[24] G. Shin, Prime ideals and sheaf representation of a pseudo symmetric ring, Trans. Amer. Math. Soc. 184 (1973), 43-60.

Jeoung Soo Cheon

Department of Mathematics

Pusan National University

PUSAN 609-735, KOREA

E-mail address: jeoungsoo@pusan.ac.kr 


\section{Chan Huh}

Department of Mathematics

Pusan National University

Pusan 609-735, Korea

E-mail address: chuh@pusan.ac.kr

Tai Keun Kwak

Department of Mathematics

DAEJIN UNIVERSITY

Pocheon 487-711, KoreA

E-mail address: tkkwak@daejin.ac.kr

YANG LEE

Department of Mathematics Education

Pusan National University

Pusan 609-735, Korea

E-mail address: ylee@pusan.ac.kr 